\title{
Influencia de las nanopartículas de sílice en polímeros termoplásticos
}

\author{
Dr. José Vega Baudrit, ${ }^{1}$ Dr. José Miguel Martín Martínez, ${ }^{2}$ \\ Quím. Melissa Camacho Elizondo 3
}

\begin{abstract}
RESUMEN: En este trabajo se prepararon sílices con distinto grado de hidrofilicidad para reducir el grado de interacción con el poliuretano y analizar la incidencia en las propiedades de los materiales compuestos obtenidos.

Se incorporó una sílice pirogénica hidrófila a adhesivos de poliuretano con distinta relación NCO/ $\mathrm{OH}$ donde el grado de separación de fases se vio favorecido en todos los poliuretanos, indicando una posible interacción de los grupos silanol de la sílice mediante enlaces de hidrógeno con el polímero. Por lo tanto, debería existir una variación de propiedades en los poliuretanos como respuesta a la presencia de la sílice dispersada.

PalABRAS CLAVE: adhesivos, poliuretanos, nanosílices, nanomateriales, nanotecnología.
\end{abstract}

ABSTRACT: In this work we have prepared silicas with different hydrophilicity to reduce the degree of interaction with the polyurethane and analyze the effect on the properties of the composites obtained.

A hydrophilic pyrogenic silica was incorporated in different polyurethane adhesives with different relation $\mathrm{NCO} / \mathrm{OH}$ where the degree of phase separation is enhanced at all polyurethanes, indicating a potential interaction of the silanol groups of the silica by hydrogen bonding with the polymer. Therefore, there should be a variation in properties as polyurethanes response to the presence of the dispersed silica.

KEY wORDS: adhesives, polyurethanes, nano silica, nanomaterials, nanotechnology.

\section{INTRODUCCIÓN}

Los poliuretanos son el producto de la condensación de un poliisocianato con un poliol, en presencia de otras sustancias. Dependiendo del uso al que se destine el poliuretano y de las propiedades deseadas, se han desarrollado varios tipos de poliuretanos. Las aplicaciones como adhesivos se derivan de la baja viscosidad y alta polaridad del material de partida. En general, se busca una buena penetración del sustrato, una fácil polimerización a temperatura ambiente, una variada estructura segmentada y una adecuada polaridad. Finalmente, todas estas características imparten a los adhesivos adecuados puntos de unión al sustrato (adhesión) y una alta fuerza cohesiva del poliuretano consigo mismo (cohesión). Los adhesivos de poliuretano son empleados en la industria de calzado en la unión suela/corte, entre otras aplicaciones.

\footnotetext{
Investigador, Laboratorio de Polímeros, POLIUNA, Universidad Nacional. Costa Rica. (jvegab@gmail.com).

2 Investigador, Laboratorio de Adhesión y Adhesivos, Departamento de Química Inorgánica, Universidad de Alicante. España.

3 Investigadora, Laboratorio Nacional de Nanotecnología, LANOTEC, Costa Rica. (Kmce08@gmail.com).
} 
Los adhesivos de poliuretano termoplásticos (TPU), basados en 4,4' difenil metano diisocianato (MDI) y poliésteres, utilizando 1,4-butanodiol como agente extendedor de la cadena, han sido ampliamente estudiados [1,2].

Los poliuretanos termoplásticos son materiales poliméricos segmentados que, por lo general, exhiben buenas propiedades mecánicas, elásticas, y de dureza, entre otras. Los TPUS usualmente exhiben una microestructura de dos fases, producto de la incompatibilidad entre los segmentos duros y blandos. Los segmentos duros (constituidos por la reacción de MDI y el extendedor de cadena) generan zonas o dominios semicristalinos, mientras que los segmentos blandos (constituidos por las cadenas de poliol) están caracterizados por ser amorfos, y están dispersos entre los segmentos duros. Estos últimos pueden actuar como puntos de entrecruzamiento físico, mientras que los segmentos blandos se comportan como la matriz flexible. Esta separación de microfases produce una mejora de las propiedades mecánicas.

El grado de separación de fases no sólo depende de la relación $\mathrm{NCO} / \mathrm{OH}$, sino también del tipo de extendedor de la cadena, peso molecular del segmento blando, formación de enlaces de hidrógeno entre los enlaces uretano, proceso de síntesis, y utilización de cargas, entre otros. Las sílices pirogénicas son un buen ejemplo de estas cargas pues presentan grupos siloxano y grupos silanol en su superficie. Los grupos siloxano son responsables del carácter elevadamente inerte de la sílice, mientras que los grupos silanol le conceden propiedades hidrofílicas [1].

En trabajos anteriores, se ha demostrado que la adición de sílices pirogénicas es de gran utilidad para mejorar sus propiedades de adhesión [1-3]. Se ha postulado la existencia de enlaces de hidrógeno entre los grupos silanol de la superficie de la sílice y los grupos uretano o hidroxilo de las cadenas de poliéster del poliuretano. Para verificar esta hipótesis, en este trabajo se han preparado sílices con distinto grado de hidrofilicidad para reducir el grado de interacción con el poliuretano y analizar la incidencia en las propiedades de los materiales compuestos obtenidos.

\section{Metodología}

En la tabla 1 se muestran las sílices estudiadas. Estas sílices fueron preparadas por Wacker-Chemie/Burghausen, Alemania, partiendo de una sílice pirogénica totalmente hidroxilada (HDK N20) y tratándola con un silano para reducir el número de grupos silanol en la superficie.

TABLA 1. Propiedades de las sílices utilizadas ${ }^{(a)}$

\begin{tabular}{|l|c|c|c|}
\hline \multicolumn{1}{|c|}{ Muestra } & $\begin{array}{c}\text { SiOH } \\
(\%)\end{array}$ & $\begin{array}{c}\text { mmol } \\
\text { SiOH/g } \\
\text { HDK }\end{array}$ & $\begin{array}{c}\mathbf{m m o l} \\
\text { SiOH/m }\end{array}$ \\
\hline HDK N20 & 100 & 0.60 & 2.99 \\
\hline HDK H20 & 57.5 & 0.34 & 1.72 \\
\hline HDK H20RD & 15.5 & 0.09 & 0.46 \\
\hline
\end{tabular}

(a) Radio primario de partícula $=7 \mathrm{~nm}$.

Fuente: Datos suministrados por Wacker-Chemie. 


\section{Caracterización de las Sílices}

La superficie específica de las sílices fue obtenida por aplicación de la ecuación BET a las isotermas de adsorción de nitrógeno a $77 \mathrm{~K}\left(-196^{\circ} \mathrm{C}\right)$. Estas isotermas se obtuvieron en un sistema Quantachrome. Las sílices se degasificaron a $100{ }^{\circ} \mathrm{C}$ durante 8 horas a $10^{-6}$ torr.

La química de las sílices se analizó mediante espectroscopia IR con transformada de Fourier. Se utilizó un espectrofotómetro Bruker Tensor 27 con una relación señal ruido de $0.04 \% \mathrm{~T}$ (a $2000 \mathrm{~cm}^{-1}$ ), en modo de transmisión, utilizando pastillas de $\mathrm{KBr}$ previamente desecado a $100^{\circ} \mathrm{C}$.

\section{SíNTESIS DE LOS POLIURETANOS}

Los reactivos utilizados han sido poliadipato de 1,4-butanodiol (Hoopol F-530, Hoocker) como poliol, el isocianato MDI (98\% de pureza, Aldrich), y 1,4-butanodiol (99\%, Aldrich) como extendedor de la cadena. Se empleó una relación NCO/OH de 1,05. Se utilizó un reactor de vidrio encamisado con un sistema de agitación mecánico a 80 rpm y la temperatura se mantuvo constante a $65^{\circ} \mathrm{C}$. La reacción se llevó a cabo durante 90 minutos, en atmósfera de nitrógeno seco. Finalizada la reacción, el poliuretano fue completamente curado (annealing) en un horno durante 12 horas a $80^{\circ} \mathrm{C}$.

\section{Preparación de los adHesivos}

Se adicionó un 20\% en peso de poliuretano, y un 2\% en peso de sílice pirogénica, utilizando 2-butanona (MEK) como disolvente. Las disoluciones de adhesivo fueron preparadas utilizando un agitador mecánico Cowles. Se mezcló la sílice con 1/3 del volumen final del disolvente a una velocidad de agitación de 2,500 rpm, durante 15 minutos. Por último, se adicionó el poliuretano y los 2/3 restantes del MEK, agitándose a 2,000 rpm durante 2 horas.

La caracterización del producto se realizó preparando películas de poliuretano exentas de disolvente. Estas películas se prepararon colocando una cierta cantidad de la disolución adhesiva en moldes de teflón de 120 x 120 mm. El disolvente se dejó evaporar en un recipiente cerrado con atmósfera saturada de MEK. El grosor de las películas obtenidas fue de aproximadamente $1 \mathrm{~mm}$ (medidas con un Vernier).

\section{CaRACTERIZACIÓN DE LOS ADHESIVOS DE TPU: CALORIMETRÍA DIFERENCIAL DE BARRIDO (DSC)}

Se utilizó un equipo TA Instrument DSC Q100 V6.2, y se determinó la temperatura de transición vítrea (Tg), la temperatura de fusión (Tm), la temperatura de cristalización (Tc), la entalpía de fusión $(\Delta \mathrm{Hm})$ y la entalpía de cristalización $(\Delta \mathrm{Hc})$ de películas de poliuretano. Se realizaron barridos de temperatura de $-80^{\circ} \mathrm{C}$ a $80^{\circ} \mathrm{C}$, empleando una velocidad de calentamiento de $5{ }^{\circ} \mathrm{C} / \mathrm{min}$. 


\section{TERMOGRAVIMETRÍA}

Se utilizó un equipo SETARAM modelo 92-16, a una velocidad de calentamiento de 10 ${ }^{\circ} \mathrm{C} / \mathrm{min}$. La temperatura se modificó entre 25 y $800^{\circ} \mathrm{C}$, en atmósfera de helio y se empleó una masa cercana a los $10 \mathrm{mg}$.

\section{Reología de platos paralelos}

Se utilizó un equipo Bohlin CS50 de esfuerzo controlado de platos paralelos de 20 mm de diámetro. Se realizó un barrido de temperaturas desde $200{ }^{\circ} \mathrm{C}$ a $30^{\circ} \mathrm{C}$ con una velocidad de enfriamiento de $5^{\circ} \mathrm{C} / \mathrm{min}$. Se usó una frecuencia de oscilación de $1 \mathrm{~Hz}$.

\section{Propiedades mecánicas}

Se utilizó el método indicado en la norma ISO 37-77. Se midieron tanto la tensión en la carga máxima como el porcentaje de deformación en la carga máxima. Se utilizó un equipo INSTRON 4411 con una velocidad de deformación de $100 \mathrm{~mm} / \mathrm{min}$ y una carga aplicada de $5 \mathrm{KN}$.

\section{ENSAYOS DE DUREZA}

Se utilizó un equipo de análisis de dureza Shore D.

\section{ENSAYOS DE PELADO EN T}

Se utilizaron probetas de PVC flexible unidas mediante adhesivos de poliuretano. Las probetas tenían una geometría rectangular de 150x30x3 mm, y antes de ser utilizadas fueron limpiadas con MEK. Se aplicó alrededor de $1 \mathrm{ml}$ de adhesivo a cada pieza de PVC y se dejó secar durante 30 minutos. El adhesivo sobre la superficie de las probetas fue reactivado utilizando radiación infrarroja a $80^{\circ} \mathrm{C}$, se pusieron en contacto las dos probetas reactivadas e inmediatamente se las sometió a una presión de $2 \mathrm{Kg} / \mathrm{cm}^{2}$ durante 10 segundos. Las fuerzas de pelado de T fueron medidas en función del tiempo transcurrido desde la realización de la unión adhesiva y se empleó una velocidad de separación de las mordazas de 100 mm/min. Se utilizó un equipo INSTRON modelo 4411.

\section{RESULTADOS Y DISCUSIÓN}

En la figura 1 se incluyen las isotermas de adsorción de algunas sílices. Las isotermas corresponden al tipo II de la clasificación BDDT (Brunauer, Deming, Deming y Teller) propia de sólidos no porosos. La condensación capilar se empieza a producir a presiones relativas cercanas a 0.85 . El tratamiento con silanos reduce tanto la capacidad adsorbente como la superficie específica de la sílice (tabla 2). De hecho, el parámetro $\mathrm{C}$-medida de la interacción o reactividad de la superficie estudiada- disminuye 
marcadamente al disminuir el contenido de grupos silanol superficiales (tabla 2), indicando una menor interacción del adsorbato con la sílice. Puesto que el tratamiento con silanos no crea porosidad en la sílice, se debe haber producido una aglomeración de las partículas de sílice durante dicho tratamiento.

TABLA 2. Parámetro $C$ y superficie específica de las sílices (ecuación de BET)

\begin{tabular}{|l|c|c|}
\hline \multicolumn{1}{|c|}{ Sílice } & C & Superficie específica $\mathbf{~ m}^{2} \mathbf{~ g ~}$ \\
\hline HDKN20 & 114 & 161 \\
\hline HDKH2ORD & 13.5 & 97 \\
\hline
\end{tabular}

Fuente: Elaboración propia.

FIGURA 1. Isotermas de adsorción de nitrógeno a 77K de algunas sílices.

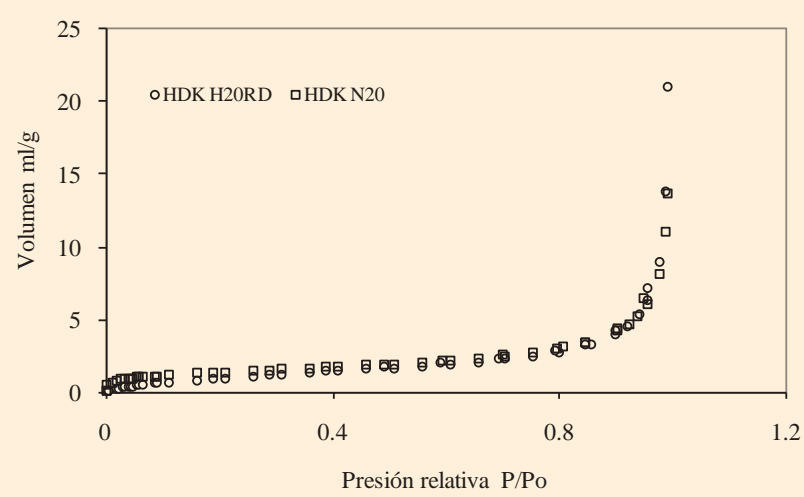

Fuente: Elaboración propia.

FIGURA 2. Espectros IR de las sílices.

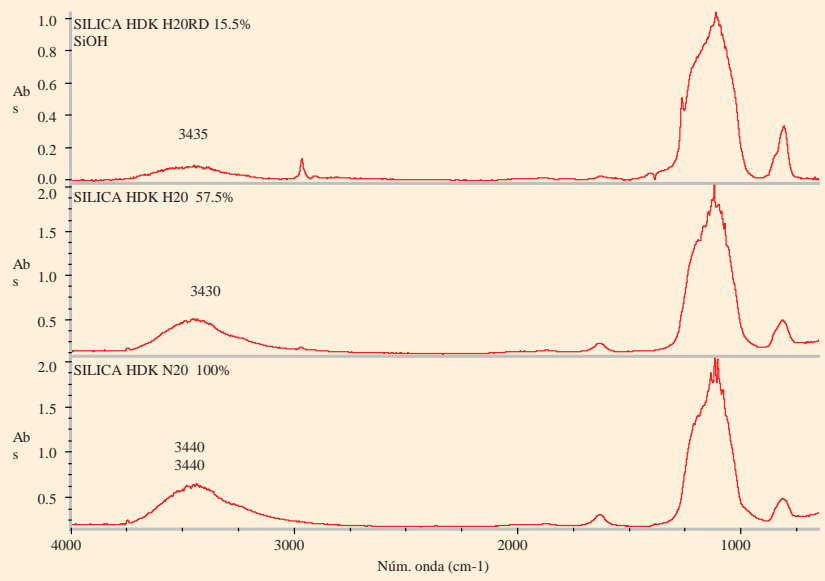

Fuente: Elaboración propia. 
La tabla 3 muestra las bandas de absorción características de las sílices. Los espectros IR de las sílices (figura 3) muestran que la banda $3430 \mathrm{~cm}^{-1}$ correspondiente a los grupos $\mathrm{OH}$ aumenta su intensidad relativa al aumentar el contenido de grupos silanol. Por otra parte, las bandas debidas a Si-O-C $\left(810,2960 \mathrm{~cm}^{-1}\right)$ aumentan su intensidad en las sílices con menor número de grupos silanol.

TABLE 3. Bandas de absorción de las sílices

\begin{tabular}{|c|c|c|}
\hline $\bar{v}\left(\mathbf{c m}^{-1}\right)$ & Grupo & Vibración \\
\hline$\sim 3440$ & SiO-H & Stretching (st) \\
\hline$\sim 2960$ & C-H & Stretching (st) \\
\hline$\sim 1630$ & SiO-H & Bending (d) \\
\hline$\sim 1260$ & Si-O, Si-H & Stretching (st), bending (d) \\
\hline $1110-1000$ & Si-O-C, Si-O-Si & Stretching (st) \\
\hline$\sim 810$ & Si-OH, Si-OC, Si-O-Si & Stretching (st) \\
\hline
\end{tabular}

Fuente: Elaboración propia.

Los valores de $\mathrm{T}_{\mathrm{g}}$ de las muestras PU-sílice obtenidos con DSC se observan en la figura 3. Se aprecia que al aumentar el grado de hidrofilicidad de la sílice incorporada al PU, el valor de la temperatura de transición vítrea aumenta debido a la interacción por enlaces de hidrógeno de los grupos superficiales de la sílice con las cadenas de los segmentos blandos del poliol.

FIGURA 3. Temperatura de transición vítrea de los poliuretanos que contienen sílices con distintos grados de hidrofilicidad.

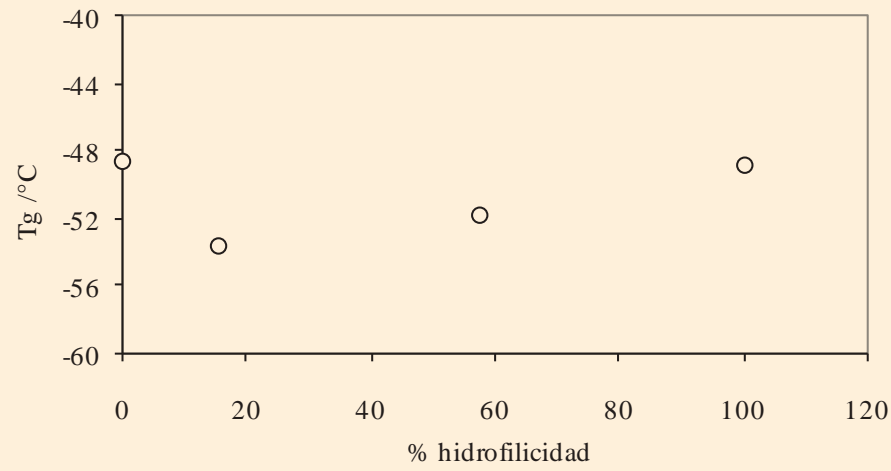

Fuente: Elaboración propia.

En las figuras 4 y 5 se observa un aumento en las entalpías de cristalización y fusión, así como de las temperaturas de cristalización y fusión, conforme se incrementa el grado de hidrofilicidad de la sílice. 
FIGURA 4. Temperatura de cristalización y entalpía de cristalización de los poliuretanos que contienen sílices con distintos grados de hidrofilicidad.

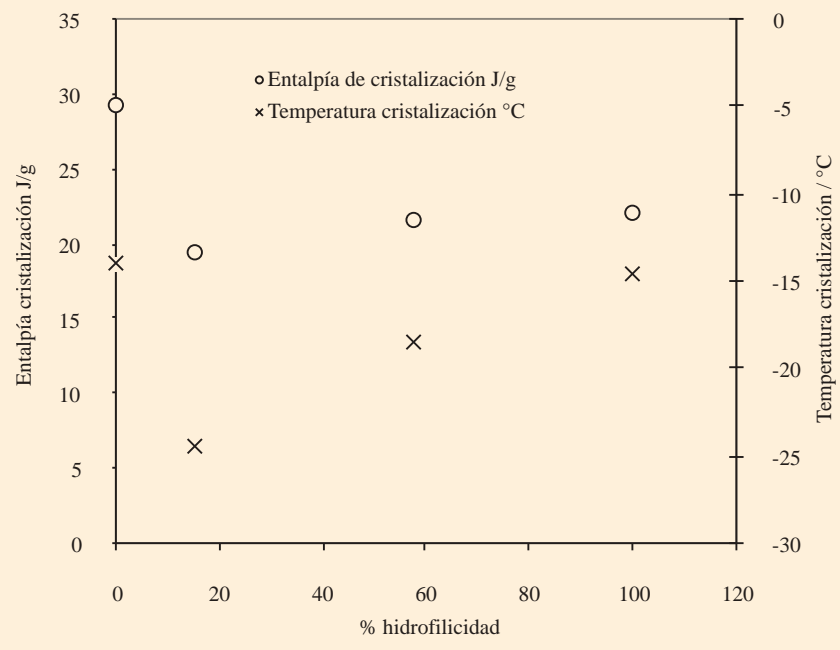

Fuente: Elaboración propia.

FIGURA 5. Temperatura de fusión y entalpía de fusión de los poliuretanos que contienen sílices con distintos grados de hidrofilicidad.

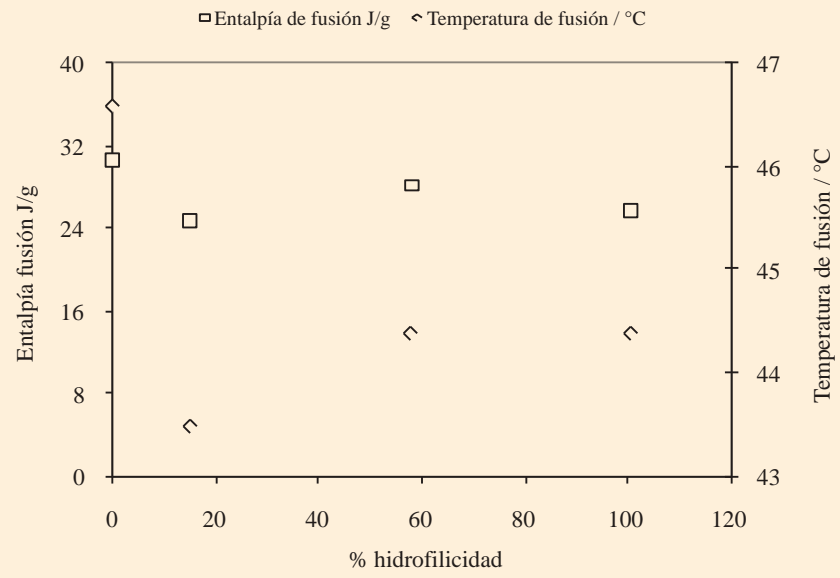

Fuente: Elaboración propia.

El análisis termogravimétrico (figura 6) muestra un considerable aumento en la temperatura a la cual el poliuretano que contiene sílice comienza a degradarse, es decir, este compuesto inorgánico actúa como un retardador de la descomposición térmica. No se observan diferencias significativas entre las muestras con diferentes sílices. En la figura 7 se observa que el material que presenta la mayor velocidad de descomposición es el que no tiene incorporado la sílice. 
FIGURA 6. Curvas TG de los poliuretanos que contienen sílices de distintos grados de hidrofilicidad.

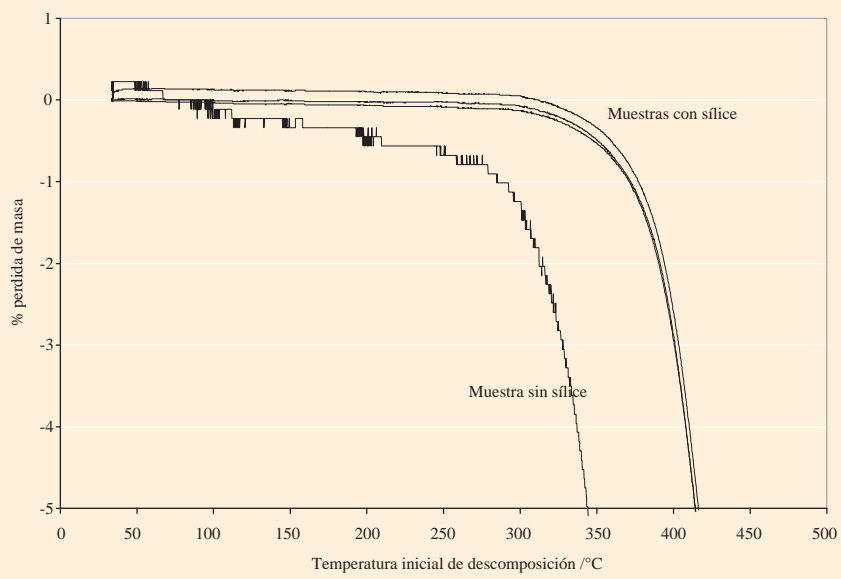

Fuente: Elaboración propia.

FIGURA 7. Temperatura de máxima velocidad de descomposición obtenida mediante análisis termogravimétrico de los poliuretanos que contienen sílices de distintos grados de hidrofilicidad.

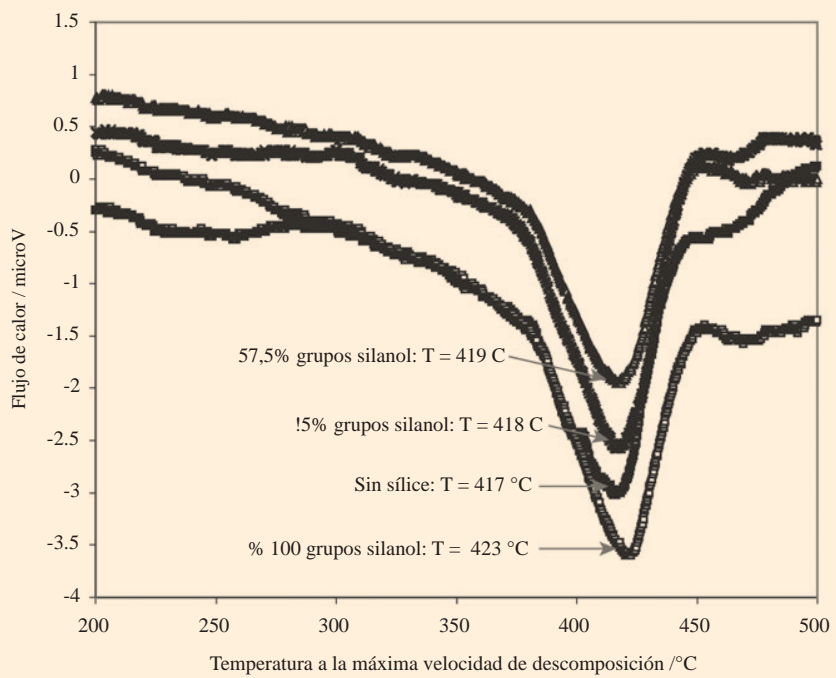

Fuente: Elaboración propia.

Con respecto a los resultados obtenidos en la reología de platos paralelos, mostrados en la figura 8, se observa un incremento tanto en el módulo de almacenamiento $\left(G^{\prime}\right)$ como de pérdida (G') en función del aumento de la hidrofilicidad de la sílice que está incorporada en el poliuretano. La curva reológica de la película de poliuretano sin sílice muestra un punto de cruce entre el módulo viscoso y elástico cercano a los $85^{\circ} \mathrm{C}$. 
FIGURA 8. Curvas reológicas obtenidas mediante reometría de platos paralelos para los poliuretanos que contienen sílices de distintos grados de hidrofilicidad.

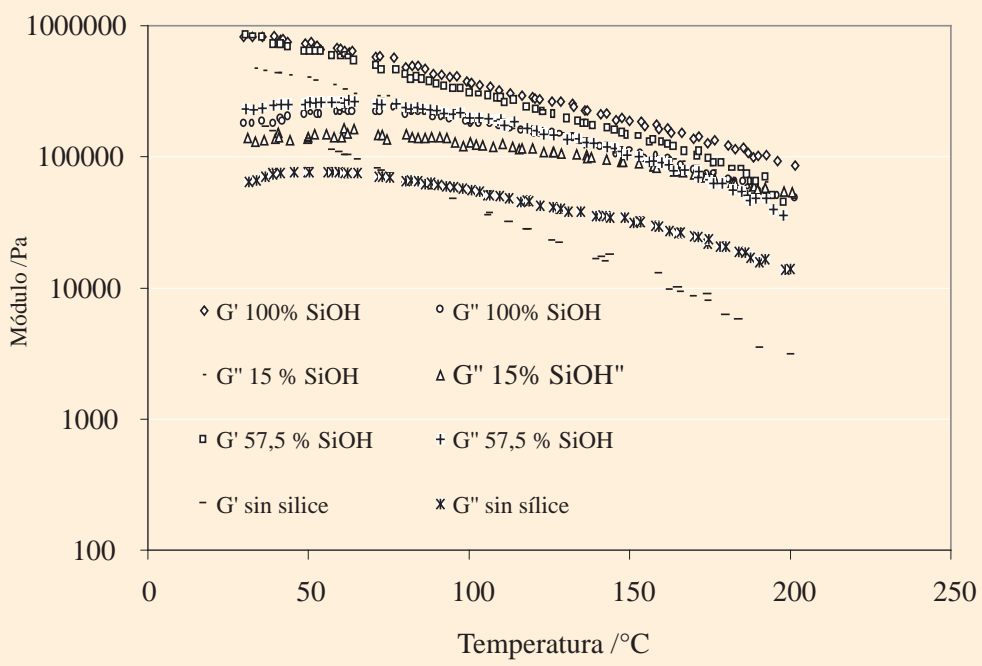

Fuente: Elaboración propia.

Si se compara la curva del material que no contiene la carga inorgánica, respecto a las demás, se observa que la adición de sílice hace desaparecer ese cruce de módulos. A mayor contenido de grupos silanol, se observa una mayor diferencia entre las curvas de G' y G", producto de la mayor interacción entre dichos grupos de la sílice y los segmentos blandos del poliuretano, lo cual, a su vez, ocasiona un aumento mayor en el módulo de almacenamiento respecto al de pérdida. En términos generales, la presencia de la sílice pirogénica, incrementa el módulo de almacenamiento y de pérdida del material, observándose un comportamiento elástico predominante

En las figuras 9a y 9b, se observan las propiedades mecánicas de los poliuretanos que contienen las diferentes sílices.

Al aumentar el grado de hidrofobicidad de la sílice se mejoran las propiedades mecánicas, pues tanto el porcentaje de deformación en la carga máxima como la tensión soportada en ese punto aumentan.

Otros grupos de investigación, utilizando un sistema similar de MDI, un poliéster derivado del ácido adípico y 1,4-butanodiol, encontraron que propiedades como el módulo de Young se incrementaron al incorporar sílices, y lo explicaron en función del aumento de la densidad de entrecruzamiento causado por las interacciones polímero-carga. Mencionan que el efecto de refuerzo depende fuertemente de la densidad de grupos silanol en la superficie de la sílice. Así, materiales con sílice de bajo contenido de grupos silanol no mostraron cambios significativos en el módulo o la dureza $[5,6]$.

Los resultados de la evaluación de la dureza se dan en la figura 10. Se observa un incremento del valor conforme se aumenta el contenido de grupo silanol de la sílice respecto al valor obtenido en el material sin sílice. Asimismo, no se observan diferencias significativas entre los valores de la dureza para los materiales que contienen 
FIGURA 9a. Evaluación de las propiedades mecánicas (porcentaje de deformación) para los poliuretanos con y sin sílice.

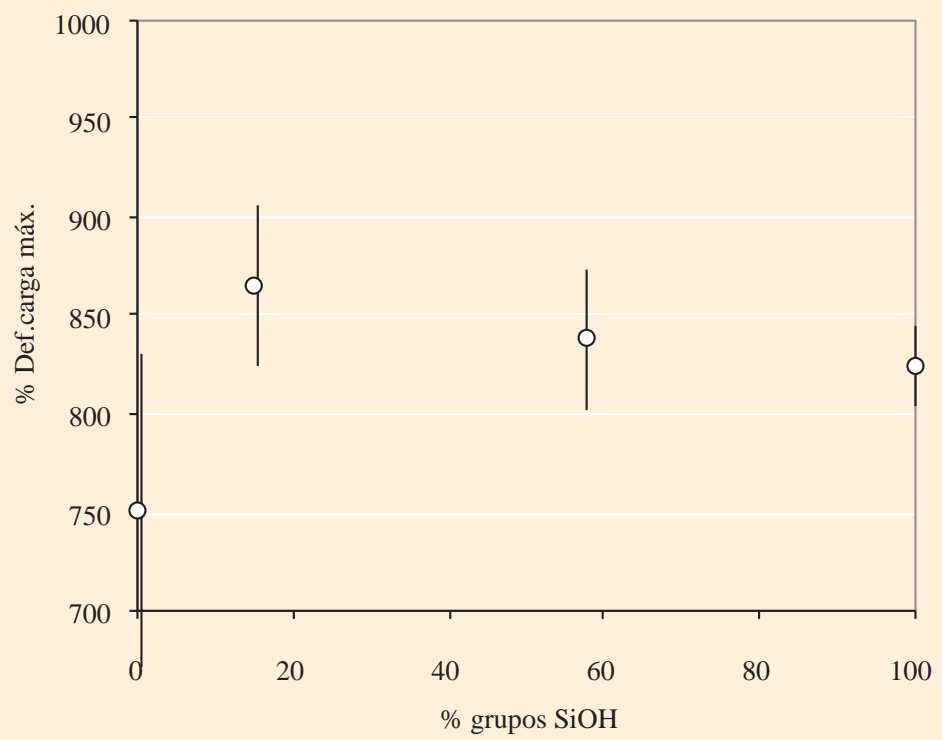

Fuente: Elaboración propia.

FIGURA 9b. Evaluación de la propiedades mecánicas (tensión en la carga máxima de deformación) para los poliuretanos con y sin sílice.

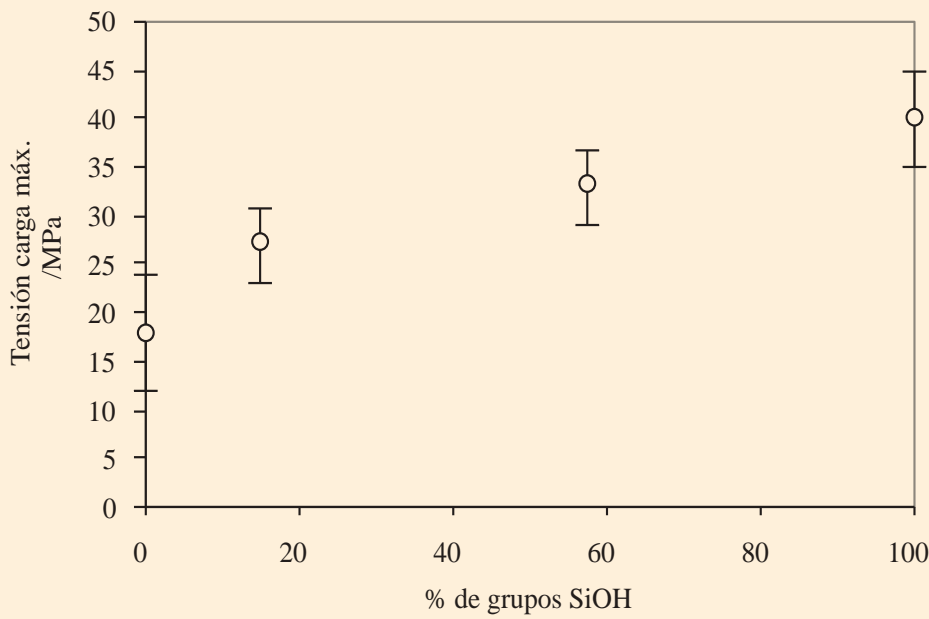

Fuente: Elaboración propia. 
diferentes sílices. Este incremento corresponde a un aumento de la cristalinidad del poliuretano [3], lo que se debe a la presencia de grupos silanol de las sílices y a su interacción con las cadenas del poliuretano.

Finalmente, los ensayos de pelado en T (figura 11) muestran un incremento en las propiedades de adhesión inicial en las uniones realizadas con los poliuretanos que contienen sílice.

FIGURA 10. Dureza Shore D para los poliuretanos con y sin sílice.

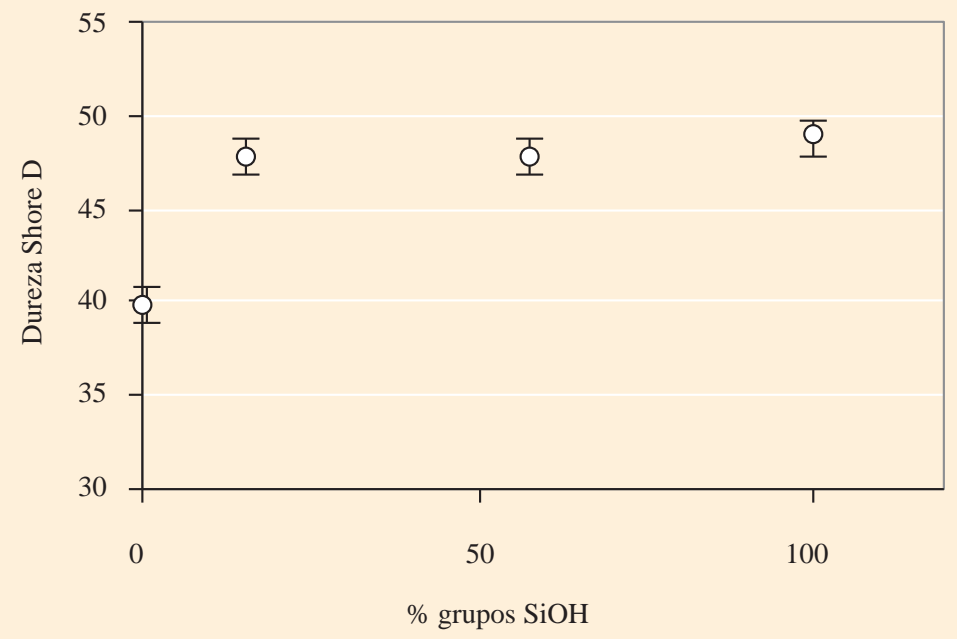

Fuente: Elaboración propia.

FIGURA 11. Resultados obtenidos en los ensayos de pelado en T de uniones PVC/adhesivo de poliuretano que contiene sílice.

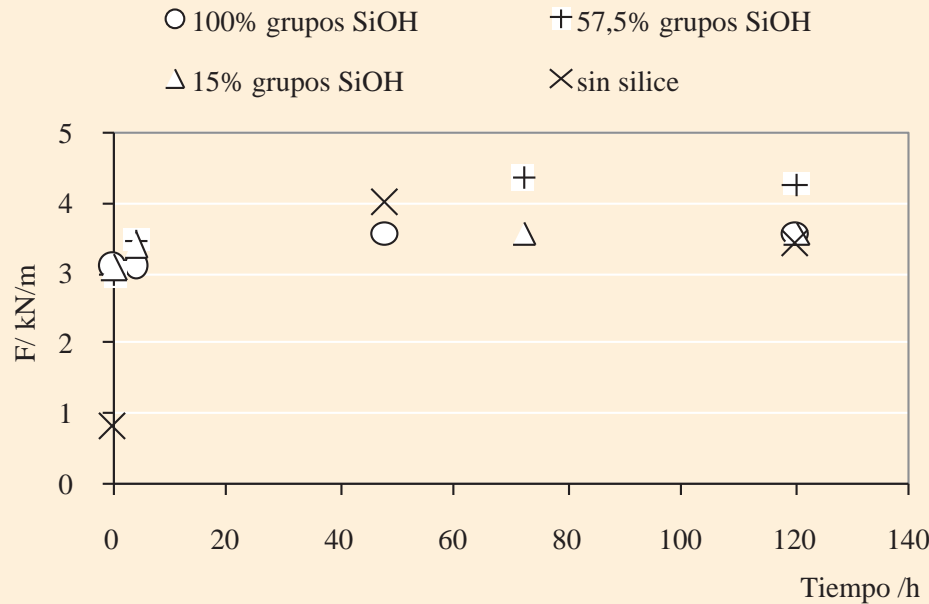

Fuente: Elaboración propia. 


\section{Conclusiones}

En términos generales, la presencia de la sílice pirogénica mejora las propiedades térmicas, mecánicas, dinámicas y de adhesión de los adhesivos de poliuretano, debido a la interacción que se produce entre la sílice y las cadenas del poliuretano, específicamente los segmentos blandos.

\section{REFERENCIAS BIBLIOGRÁFICAS}

[1] Macía-Agulló, T.G., J.C. Fernández-García, N. Pastor-Sempere y A.C. Orgilés-Barceló (1992) "Addition of silica to polyurethane adhesives". J. Adhesion, 38, 31-53.

[2] Ferrándiz-Gómez, T.P., J.C. Fernández-García, A.C. Orgilés-Barceló y J.M. Martín-Martínez (1996) J Adhes Sci Technol., 10, 833.

[3] Oertel, G. (1985) Polyurethane handbook. New York: Hanser Publisher.

[4] Pastor-Sempere, N., J.C. Fernández-García, A.C. Orgilés-Barceló y J.M. Martín-Martínez (1995) Fumaric acid as a promoter of adhesion in vulcanized synthetic rubbers. J Adhesion, 50, 25-42.

[5] Nunes, R.C.R., J.L.C. Fonseca y M.R. Pereira (2000) Polymer-filler interactions and mechanical properties of a polyurethane elastomer. Polymer Testing, 19, 93-103.

[6] Nunes, R.C.R., R.A. Pereira, J.L.C. Fonseca y M.R. Pereira (2001) "X-ray studies on compositions of polyurethane and silica". Polymer Testing, 20, 707-712. 